\title{
Relación entre ansiedad matemática y rendimiento académico en matemáticas en estudiantes de secundaria
}

\author{
Relationship between mathematical anxiety and academic performance in \\ mathematics in high school students
}

\section{Relação entre ansiedade matemática e rendimento acadêmico em matemática em estudante de ensino médio}

\author{
Gustavo Villamizar Acevedo ${ }^{1}$, ORCID 0000-0002-8886-1993 \\ Tammi Yulien Araujo Arenas ${ }^{2}$, ORCID 0000-0003-4681-5566 \\ Wenddy Jurany Trujillo Calderón ${ }^{3}$, ORCID 0000-0002-1952-8150 \\ ${ }^{12}$ Universidad Pontificia Bolivariana - Bucaramanga. Colombia \\ ${ }^{3}$ Corporación Dignificar - Floridablanca. Colombia
}

\begin{abstract}
Resumen: Esta investigación correlacional buscó identificar la relación entre ansiedad matemática y rendimiento académico en matemáticas. Se trabajó con 127 estudiantes de un colegio de secundaria de Colombia, 68 niñas y 59 niños, con edad promedio de 14.34 años, quienes respondieron la Escala de ansiedad matemática de Fennema-Sherman, la cual tiene un nivel de confiabilidad de .88. Los resultados mostraron que el promedio académico es 4.1, alto según los criterios establecidos por el Ministerio de Educación Nacional de Colombia, que las niñas tienen un promedio más alto y que su nivel de ansiedad es mayor que los niños. En la relación ansiedad matemática vs. rendimiento académico en matemáticas, se halló una relación inversa, es decir a mayor ansiedad menor rendimiento académico. Como conclusión se propuso a las directivas del colegio diseñar un programa en pro de la modificación de respuestas emocionales, debido a que se evidenció en las niñas, la influencia de factores afectivos en el aprendizaje de las matemáticas.
\end{abstract}

Palabras clave: ansiedad matemática, estudiante de secundaria, matemáticas, relación, rendimiento académico

Abstract: The objective of this correlational research is to identify the relation between mathematical anxiety and academic performance in the area of mathematics by analyzing the answers to the Fennema-Sherman mathematical anxiety scale (.88 confidence level) given by 127 secondary school students (68 girls and 59 boys) with an average age of 14.34 years old. The GPA of students was 4.1, a high GPA according to the criteria of the Colombian Ministry of National Education. Furthermore, girls obtained a higher GPA than boys although their level of anxiety was higher too. Regarding the relation between mathematical anxiety and academic performance in the area of mathematics, this study shows that they are inversely related, that is, the greater the anxiety, the lower the academic performance. As a result of this study, the staff of school directors were suggested to design a program to modify the emotional responses of students, considering the fact that girls are influenced by affection-related factors with regard to the mathematical learning process.

Keywords: Mathematical anxiety, secondary education students, mathematics, relation, academic performance 
Resumo: Esta pesquisa correlacional buscou identificar a relação entre ansiedade matemática e rendimento acadêmico em matemática. Trabalhou-se com 127 estudantes de um colégio de ensino médio da Colômbia, 68 meninas e 59 meninos, com idade média de 13.34 anos, que responderam à Escala de ansiedade matemática de Fennema-Sherman, cujo nível de confiabilidade é de .88. Os resultados demonstraram que a média acadêmica é 4.1, considerada alta de acordo com os critérios estabelecidos pelo Ministério de Educação Nacional da Colômbia; que as meninas têm uma média mais alta; e que seu nível de ansiedade é maior que o dos meninos. Em relação à ansiedade matemática vs. rendimento acadêmico em matemática, foi encontrada uma relação inversa, ou seja, quanto maior a ansiedade, menor o rendimento acadêmico. Como conclusão, se propõe à direção do colégio projetar um programa a favor da modificação das respostas emocionais, devido ao fato de a influência de fatores afetivos no aprendizado da matemática ser evidente nas meninas.

Palavras-chave: ansiedade matemática, estudante de ensino médio, matemática, relação, rendimento acadêmico

Recibido: $24 / 07 / 2019$

Aceptado: 27/04/2020

Cómo citar este artículo:

Villamizar Acevedo, G., Araujo Arenas, T.Y., Trujillo Calderón, W.J. (2020). Relación entre ansiedad matemática y rendimiento académico en matemáticas en estudiantes de secundaria. Ciencias Psicológicas, 14(1), e-2174. doi: https://doi.org/10.22235/cp.v14i1.2174

Correspondencia: Gustavo Villamizar Acevedo. Grupo de investigación Análisis y Transformación Psicosocial. Universidad Pontificia Bolivariana Bucaramanga, Colombia. E-mail: gustavo.villamizar@upb.edu.co Tammi Yulien Araujo Arenas, e-mail: yulien_196@hotmail.com. Wenddy Jurany Trujillo Calderón. Corporación Dignificar. Floridablanca.Colombia.E-mail: wenddy25@outlook.com

\section{Introducción}

Como resultado de un gran número de investigaciones realizadas a nivel mundial que comprueban la existencia de dificultades emocionales frente a las matemáticas en personas de diversas edades y niveles de formación, se acuñó el término ansiedad matemática.

Este constructo, se originó en un estudio de Dreger y Aiken, quienes evidenciaron que muchos estudiantes universitarios mostraban malestar ante el pedido de resolver problemas matemáticos, reacción que denominaron ansiedad ante los números y refirieron que los estudiantes no presentaban ansiedad generalizada, pero sí un tipo de ansiedad específica relacionada con la aplicación del cálculo y otras operaciones numéricas (Dos Santos \& Morales, 2012).

Una vez detectada esta problemática y asumida su denominación como ansiedad matemática se generaron una serie de definiciones (Pérez-Tyteca, Monje \& Castro, 2013; Reali, Maldonado \& Jiménez, 2015; Richardson \& Suinn, 1972), considerándola como una reacción emocional intensamente negativa (tensión, nervios, miedo, preocupación, inquietud, irritabilidad, impaciencia, confusión, bloqueo mental) que dificulta la resolución de problemas matemáticos, tanto cotidianos como académicos, e incrementa la autopercepción de incompetencia en tareas de esta naturaleza.

En la anterior definición se expresa que la ansiedad matemática, es una situación especial que se presenta cuando se conjugan factores de personalidad, ambientales e intelectuales. Entre los factores personalidad se encuentran la baja autoestima y el temor para 
preguntar; entre los ambientales, las experiencias negativas en el aprendizaje de las matemáticas, y las actitudes negativas tanto de padres de familia como de profesores; y entre los intelectuales, la sensación de incompetencia para aprender matemáticas, la falta de utilidad percibida de las matemáticas y la no coordinación entre los estilos de enseñanza de los profesores y los estilos de aprendizaje de los estudiantes (Jaggernauth \& Jameson-Charles, 2010).

Respecto a las consecuencias que este fenómeno crea se encuentran: conductas de evitación, por ello los estudiantes tienden a seleccionar campos de formación que no tenga en sus itinerarios áreas de conocimiento relacionadas con las matemáticas; afianzamiento de actitudes negativas hacia todo que pueda asociar con el cálculo y la aritmética, así como afectación negativa de la motivación y la autoconfianza matemática (Palacios, Hidalgo, Maroto \& Ortega, 2013) y deficiencias en el rendimiento académico.

En lo correspondiente a la relación ansiedad matemática-rendimiento académico, Reyes (1984) afirmó que los estudios en este campo han mostrado desde hace décadas, la presencia de correlaciones negativas significativas: entre mayor sea la ansiedad matemática menor será el rendimiento.

Según Ashcraft y Kirk (2001) los estudiantes con alta ansiedad matemática a la hora de afrontar una tarea también se enfrentan a sus preocupaciones, miedos y angustias, situación que afecta su rendimiento generando resultados deficientes, hecho que le impide notar sus capacidades para abordar exitosamente los contenidos de esta disciplina.

Hidalgo, Maroto y Palacios (2004, p. 93) identificaron una cadena en la cual participa la ansiedad como factor desencadenante en la dificultad para alcanzar logros académicos en la matemática. Dicha cadena la describen de la siguiente manera:

la dificultad intrínseca y acumulativa de las Matemáticas produciría en el devenir escolar alumnos con lagunas importantes que desembocan, más tarde o más temprano, en unos rendimientos escolares insatisfactorios, lo que determina una disminución progresiva del autoconcepto matemático y atribuciones de causalidad negativas (fatalistas) a la par que una desgana que genera aburrimiento y rechazo que, no sólo no ayuda, sino que empeora la comprensión de la asignatura que es percibida, de año en año, como un tormento.

Esta situación llevó a algunos académicos a indagar sobre este fenómeno, tanto en estudiantes universitarios como en educación media. En el primer estudio realizado sobre el tema, los investigadores Dreger y Aiken predijeron la existencia de una relación inversa entre ansiedad matemática y rendimiento académico (Ashcraft \& Moore, 2009). Entre las investigaciones que corroboraron este aserto, tanto en escolares como en universitarios se encuentran las realizadas en Colombia, México y Perú por Reali et al. (2015), Serrano y Sánchez (2017), y Castro (2017). Por otra parte, Agüero, Meza, Suárez y Schmidt (2017), evidenciaron que el $78 \%$ de los estudiantes de educación media de Costa Rica muestra ansiedad matemática, que las mujeres son más ansiosas que los hombres, y que en la medida que se sube en el nivel de formación la ansiedad matemática se incrementa.

Una explicación ante los resultados de diversas investigaciones que relacionan bajo rendimiento académico en matemáticas con niveles no deseados de ansiedad, es que ella es producto del accionar de los estudiantes y lleva a quien vive esta problemática a restarle valor al dominio académico y centrarse más en sus dificultades personales y en los fallos previos (Rivas, 1997).

Pero no todos los investigadores asumen que la ansiedad ante la matemática genera bajo rendimiento, Guerrero y Blanco (2004) explican esta afirmación a partir de la hipótesis de la «U invertida» o teoría de la zona de funcionamiento óptimo, según la cual la existencia de un nivel idóneo de ansiedad produciría niveles óptimos de rendimiento.

Respecto al rendimiento académico de los escolares colombianos en el área de las matemáticas, los resultados alcanzados en pruebas masivas o estandarizadas como PISA o las estatales (Saber) son deficientes. En la prueba Pisa, de todas las áreas evaluadas, los resultados 
en el área de matemáticas fueron los peores, solo el 1\% se ubicaron en el Nivel 5 o superior en matemáticas (OECD, 2019). En las Pruebas Saber, el promedio de los resultados, generalmente ha estado por debajo de la media (Mesa, 2016).

Las razones dadas para explicar estos bajos logros son variadas, Linares (2013) la asocia, entre otras, con un supuesto "odio" que los estudiantes sienten hacia las matemáticas y al mito que son difíciles. La OECD (2019) vincula estos resultados con el nivel socioeconómico, que según esta agencia explica el $13 \%$ de la variación en rendimiento en matemáticas, y con género (los chicos superaron a las chicas en matemáticas por 20 puntos). Fernández, Hernández, Prada y Ramírez (2018), agregan elementos vinculados a las prácticas pedagógicas, el dominio afectivo, las concepciones sobre las Matemáticas y las actuaciones de los docentes. En lo relacionado con el dominio afectivo involucran la ansiedad ante unos contenidos que los estudiantes consideran de difícil comprensión.

La situación descrita lleva a plantear el siguiente problema: ¿Existe relación entre el rendimiento académico en matemáticas, ansiedad matemática y género en estudiantes de una institución de educación secundaria de Colombia?

A partir de este problema emergió el interés por realizar la siguiente investigación con el objetivo de identificar la relación entre ansiedad frente a las matemáticas y rendimiento académico, el nivel de ansiedad matemática y la existencia de diferencias entre ansiedad y rendimiento según género 1en estudiantes de bachillerato de una institución educativa privada de Colombia, en la cual el rendimiento de sus estudiantes en las pruebas Saber 11, en el año 2017 en los ranking de clasificación se encontraba en la categoría B, porque alrededor del $55 \%$ de sus estudiantes se ubicaba en la prueba de matemáticas en el 33\% inferior.

\section{Metodología}

Estudio empírico-analítico, de tipo correlacional, que pretende encontrar la existencia o no de relación entre dos variables continuas: puntaje en una prueba de ansiedad matemática y rendimiento académico en matemáticas.

\section{Población}

La población fue 135 estudiantes de bachillerato de un colegio privado de Colombia matriculados en el año 2018.

\section{Muestra}

La muestra estuvo conformada por 127 estudiantes, que corresponden al $94.07 \%$ de los estudiantes matriculados en el año 2018. De la totalidad 68 (54\%) eran mujeres y 59 (46\%) hombres. Se nota que es mayor la cantidad de mujeres datos que coinciden con las cifras nacionales y departamentales, donde la proporción de mujeres que estudian básica secundaria y media es mayor que los hombres. En cuanto a la edad la media fue 14.34, con una desviación de 164.

\section{Criterios de inclusión}

Para que los estudiantes pudieran participar en la investigación se tuvo en cuenta que se encontraran matriculados en el colegio, que no tuvieran dificultades de aprendizaje, que el docente de la asignatura de matemáticas fuera el mismo, que no reciban clases aparte de las dadas en el colegio, que sus padres o acudientes aceptaran su participación mediante un consentimiento y que los estudiantes firmaran su asentimiento. 


\section{Instrumentos}

Se trabajó con la escala de ansiedad matemática de Fennema-Sherman, y una matriz de rendimiento académico en matemáticas.

La Escala de ansiedad matemática de Fennema-Sherman es una prueba tipo Likert, que tiene una confiabilidad de .87 , consta de tres dimensiones (ansiedad global hacia las matemáticas, ansiedad hacia la resolución de problemas y ansiedad ante los exámenes) y 12 ítems con 5 opciones de respuesta que van del totalmente en desacuerdo (1) al totalmente de acuerdo (5).

Entre los ítems de la primera dimensión se encuentra el siguiente: tengo miedo a las matemáticas, de la segunda cuando hago problemas de matemáticas se me queda la mente en blanco y no soy capaz de pensar claramente y de la tercera casi siempre me pongo nervioso en un examen de matemáticas (Sánchez, Segovia \& Miñan, 2011).

El baremo, con el cual se trabajó que se presenta en la Tabla 1, fue elaborado por PérezTyteca (2012).

Tabla 1

Baremo de la escala de ansiedad matemática de Fennema-Sherman según Pérez-Tyteca

\begin{tabular}{cc}
\hline PUNTUACIÓN PROMEDIO & NIVEL DE ANSIEDAD \\
\hline Menor de 2 & Muy bajo \\
Entre 2 y 2.9 & Bajo \\
Entre 3 y 3.9 & Alto \\
Mayor de 4 & Muy alto \\
\hline
\end{tabular}

Fuente: Pérez-Tyteca, P. (2012)

En cuanto al rendimiento académico se realizó una la matriz donde se recogieron las notas obtenidas por cada estudiante durante cuatro períodos en la asignatura de matemáticas. Las notas oscilan entre 0.1 y 5.0, siendo la mínima aprobatoria de 3.5, parámetro determinado por la institución. En la investigación se asume como rendimiento el promedio académico de cada estudiante en matemáticas, resultante de la suma de cuatro notas dividida por cuatro. En cuanto a la escala de valoración toma la estipulada por el Ministerio de Educación Nacional (2009) en el Decreto 1290, adoptando como criterio de aprobación lo siguiente:

- Desempeño Superior: 4.6 - 5.0 (el estudiante tiene un excelente rendimiento académico)

- Desempeño Alto: 4.0 - 4.5 (el estudiante tiene buen rendimiento académico)

- Desempeño Básico: 3.5 - 3.9 (el estudiante pasa con lo mínimo)

- Desempeño Bajo: 0.1 - 3.4 (el estudiante pierde)

\section{Procedimiento} seis fases:

Para cumplir con los objetivos planteado al inicio de la investigación, se llevaron a cabo

Primera fase: entrega de la carta de solicitud de permiso al rector de la institución educativa.

Segunda fase: Por medio del docente de matemáticas, se entregó a cada estudiante un consentimiento informado por el cual los padres de familia o los acudientes autorizan la participación del menor en la investigación, así como la entrega de las notas por parte de la institución.

Tercera fase: entrega a cada estudiante del asentimiento informado, por el cual ellos deciden su participación en la investigación. 

del mismo.

Cuarta fase: explicación del instrumento e instrucciones para dar inicio a la aplicación

Quinta fase: elaboración de la base de datos, a partir de los resultados tanto en la prueba total como en cada una de las subpruebas y el promedio de las notas obtenidas en el área de matemáticas, con base en ellas se realizaron los procedimientos estadísticos, utilizado el software Sigmastat 3. Para determinar la prueba estadística a utilizar, inicialmente se identificó la distribución de los datos. Para encontrar el índice de correlación entre ansiedad ante la matemática-rendimiento académico, se empleó la rho de Sperman por no presentarse una distribución normal. Para identificar las diferencias según nivel de ansiedad y género, en primera medida se determinó el comportamiento de los datos en la prueba total, luego en cada una de las dimensiones. Teniendo en cuenta que tanto en la totalidad de la prueba como en las dimensiones ansiedad global y resolución de problemas no se presentó distribución normal, para identificar la existencia o no de diferencias se utilizó la prueba U de Mann-Whitney. En la dimensión exámenes se utilizó la t de students debido a que la prueba de homoscedasticidad mostró que la distribución era normal.

Sexta fase: Análisis y descripción de los resultados.

\section{Resultados}

\section{Rendimiento académico}

El promedio académico de la muestra en la asignatura de matemáticas en el año lectivo de 2018 corresponde a 4.1, con una desviación 0.05. La nota mínima fue de 2.8 y la máxima 5.0. El promedio se encuentra en el rango de desempeño alto.

En cuanto al rendimiento académico según género, el promedio en los hombres fue de 3.9 (desviación 0.08) y el de las mujeres 4.25 (desviación 0.07).

\section{Ansiedad ante las matemáticas}

La media arrojada por la prueba corresponde a 2.53, la cual según el baremo asumido es baja, ya que se encuentra entre los valores establecidos de 2 y 2.9. La desviación es de 0.80. En cuanto la distribución de los sujetos según el nivel de ansiedad, los resultados que se presentan en la Tabla 2 muestra que el porcentaje mayor se encuentra con un bajo nivel de ansiedad, le siguen en igual proporción muy bajo y alto.

Tabla 2

Distribución de la totalidad de los sujetos según nivel de ansiedad

\begin{tabular}{cccccccccc}
\hline Nivel de & \multicolumn{2}{l}{ Muy alto } & \multicolumn{2}{c}{ Alto } & \multicolumn{2}{c}{ Bajo } & \multicolumn{2}{c}{ Muy Bajo } & \multirow{2}{*}{ Total } \\
\cline { 2 - 10 } ansiedad & $\mathrm{N}$ & $\%$ & $\mathrm{~N}$ & $\%$ & $\mathrm{~N}$ & $\%$ & $\mathrm{~N}$ & $\%$ & \\
Sujetos & 5 & 3.93 & 32 & 25.19 & 58 & 45.66 & 32 & 25.19 & 127 \\
\hline
\end{tabular}

Teniendo en cuenta que la escala de ansiedad utilizada se divide en tres dimensiones se procedió a identificar los resultados en cada una de ellas. La media a nivel general por cada una de las dimensiones, tal y como se muestra en la Tabla 3 deja ver que la más alta de ansiedad corresponde a la relacionada con los exámenes.

Tabla 3

Distribución de la totalidad de los sujetos según dimensión de ansiedad ante las matemáticas 


\begin{tabular}{cccccc}
\hline ANSIEDAD & $N$ & Mínimo & Máximo & Media & Desviación \\
\hline Global & 127 & 1.0 & 4.8 & 2.146 & 0.817 \\
Resolución & 127 & 1.0 & 4.7 & 2.607 & 0.893 \\
problemas & 127 & 1.0 & 5.0 & 2.811 & 1.073 \\
Exámenes & & &
\end{tabular}

\section{Ansiedad ante las matemáticas y género}

En lo correspondiente al nivel de ansiedad matemática según género, los resultados muestran, de acuerdo con la tabla 4, que el mayor nivel se presenta, en ambos géneros, ante los exámenes.

Tabla 4

Distribución de los sujetos según género en cada dimensión de ansiedad ante las matemáticas

\begin{tabular}{|c|c|c|c|c|c|c|c|c|c|c|}
\hline \multirow{3}{*}{ ANSIEDAD } & \multicolumn{10}{|c|}{ GÉNERO } \\
\hline & \multicolumn{5}{|c|}{ FEMENINO } & \multicolumn{5}{|c|}{ MASCULINO } \\
\hline & $\mathrm{N}$ & Mín & Máx. & Media & Des. & $\mathrm{N}$ & Mín. & Máx. & Media & Des. \\
\hline Global & & 1.0 & 4.8 & 2.27 & .9006 & & 1.0 & 3.5 & 2.022 & .6963 \\
\hline $\begin{array}{l}\text { Resolución } \\
\text { problemas }\end{array}$ & 68 & 1.0 & 4.7 & 2.804 & .9190 & 59 & 1.0 & 4.3 & 2.410 & .8199 \\
\hline Exámenes & & 1.0 & 5.0 & 3.051 & 1.1004 & & 1.0 & 4.7 & 2.571 & .9905 \\
\hline
\end{tabular}

Conocido el resultado por género en las dimensiones, donde se nota mayor ansiedad en las mujeres, se buscó determinar si esas diferencias son estadísticamente significativas, para ello previamente se identificó la distribución de los datos, los resultados muestran que no se presenta distribución normal para ansiedad global y solución de problemas (ver Tabla 5), por ello para determinar la diferencia de medias de utilizó la prueba U de Mann-Whitney.

Tabla 5

Prueba de normalidad de los datos en la escala de ansiedad ante las matemáticas

\begin{tabular}{lcccccc}
\hline \multirow{2}{*}{ PRUEBA Y SUBPRUEBAS } & \multicolumn{3}{c}{ KOLMOGOROV-SMIRNOV } & \multicolumn{3}{c}{ SHAPIRO-WILK } \\
\cline { 2 - 7 } & Estadístico & $g l$ & Sig. & Estadístico & $g l$ & Sig. \\
\hline Prueba total & .075 & 127 & .073 & .983 & 127 & .106 \\
Ansiedad resolución & .118 & 127 & .000 & .969 & 127 & .005 \\
problemas & .114 & 127 & .000 & .947 & 127 & .000 \\
Ansiedad global & .082 & 127 & .036 & .967 & 127 & .004 \\
Ansiedad ante exámenes & & &
\end{tabular}

Los resultados para ansiedad global muestran que no existen diferencias significativas en los géneros. En lo correspondiente a ansiedad ante solución de problemas, los datos, que se presentan en la Tabla 6, evidencian que la ansiedad ante este tipo de situación es mayor en las niñas que en los niños y que dicha diferencia es significativa al .05. En cuanto a la ansiedad ante los exámenes, lo arrojado por la prueba de homoscedasticidad mostró que su distribución es normal. Los resultados se presentan en la tabla 5, revelan la existencia de diferencias 
significativas según género. Los resultados evidencian que las niñas presentan más ansiedad ante la solución de problemas y la presentación de exámenes.

Tabla 6

Diferencias según género en cada dimensión

\begin{tabular}{cccccc}
\hline Subprueba & Género & $\begin{array}{c}\text { Distribución } \\
\text { datos }\end{array}$ & Prueba usada & $t$ & Sig. \\
\hline $\begin{array}{c}\text { Ansiedad } \\
\text { global }\end{array}$ & $\begin{array}{c}\text { Masculino } \\
\text { Femenino }\end{array}$ & No normal & Mann-Whitney & 3483 & .156 \\
\hline $\begin{array}{c}\text { Solución } \\
\text { problemas }\end{array}$ & $\begin{array}{c}\text { Masculino } \\
\text { Femenino }\end{array}$ & No normal & Mann-Whitney & 3271 & .014 \\
\hline $\begin{array}{c}\text { Ansiedad } \\
\text { exámenes }\end{array}$ & $\begin{array}{c}\text { Masculino } \\
\text { Femenino }\end{array}$ & Normal & t student & -2.569 & .011 \\
\hline
\end{tabular}

Sig. .05

\section{Correlación ansiedad ante la matemáticas-rendimiento académico}

En la correlación notas-prueba total, los resultados presentados en la Tabla 7 muestran la existencia de una correlación negativa significativa al .05. tanto en la prueba total como en cada una de las dimensiones. lo que permite suponer que en la medida que la ansiedad ante las matemáticas aumenta el rendimiento académico en matemáticas disminuye.

Tabla 7

Correlación promedio notas vs escala total y dimensiones

\begin{tabular}{|c|c|c|c|}
\hline CORRELACIÓN & $N$ & Rho de Sperman & Sig. \\
\hline Notas Vs. Escala total & \multirow{4}{*}{127} & $-.337 *$ & .000 \\
\hline $\begin{array}{l}\text { Notas Vs. Ansiedad } \\
\text { Exámenes }\end{array}$ & & $-.247 *$ & .005 \\
\hline Notas Vs. Ansiedad Global & & $-.321 *$ & .000 \\
\hline $\begin{array}{c}\text { Notas Vs. Solución } \\
\text { problemas }\end{array}$ & & $-.317 *$ & .000 \\
\hline
\end{tabular}

Sig. .05

\section{Discusión}

Respecto al promedio académico, de acuerdo con las evaluaciones del docente, los resultados son disonantes en relación a los reportados por los informes de las evaluaciones realizadas por organismos nacionales e internacionales como el Instituto Colombiano para la Evaluación de la Educación (ICFES) y el Programa Internacional para la Evaluación de Estudiantes (PISA). Estos hallazgos se pueden explicar al comparar tanto la estructura como los fines de los instrumentos de evaluación. Las pruebas masivas están realizadas de acuerdo a parámetros psicométricos y consideran la existencia de un conocimiento único independiente del contexto o cultura (Sánchez, 2017). Además, son terminales y de carácter acumulativo. Por su parte las pruebas de aula (internas), realizadas por los docentes, tienen un carácter formativo. La 
calificación es el resultado de un conjunto de actividades, entre las cuales se encuentran exámenes, talleres, exposiciones y otras.

La situación descrita muestra diferencias fundamentales entre las pruebas masivas y las internas, que no las hace compatibles, entre la cual se destaca que las evaluaciones masivas son únicas, terminales, acumulativas, descontextualizadas, que tienen como objetivo evaluar el sistema educativo y no los estudiantes. En contraste las pruebas de aula, son de carácter formativo, se realizan de forma continua, son variadas y se centran en los estudiantes.

$\mathrm{Al}$ dividir la muestra según género se notó que el rendimiento en matemáticas fue mayor en las niñas y que dichas diferencias son estadísticamente significativas, resultado que va en contravía por los reportados en otras investigaciones como las realizadas Álvarez (2012) y Barrera, Gutiérrez, Lara y Villalpando (2011). Investigadores como Caprile, Vallès, Potron, Crèixams y Arasanz (2008) han planteado que la diferencia entre hombres y mujeres, donde generalmente los hombres obtienen mejores puntajes, responden a una multiplicidad de factores. Algunos los ligan con aspectos sociales (Caciá, Reyes, Rosales \& Marroquín. 2012; Radovic, 2018), otros a estereotipos (Gamboa, 2012; Mendick, 2005). Las razones por las cuales se dieron estos resultados son desconocidas, pero alrededor de ello se puede suponer la influencia de factores motivacionales y expectativas en las niñas, así como el clima del aula.

En torno a ansiedad matemática, los resultados muestran que la mayor proporción de la muestra presenta una ansiedad baja, lo cual coincide con lo encontrado en estudiantes de educación media costarricense por Agüero et al. (2017), datos que al igual de lo que dicen estos investigadores llaman la atención, porque alrededor de ella existe una serie de creencias sobre su dificultad que llevan a "odiarla", como resalta Rius (2015) quien afirma que las matemáticas gozan de mala fama y rechazo entre los estudiantes y González, Núñez, González, González y Roces (2003) quienes detectaron déficit motivacional y actitudes negativas hacia las matemáticas.

Una posible razón que explique estos resultados tiene que ver con que los estudiantes participantes de la investigación han tenido el mismo profesor en dos los últimos años, situación que puede llevar a no considerar el contexto como amenazador. Esta disquisición se plantea como una hipótesis, ya que de acuerdo con Martínez (2010) algunos profesores y estudiantes consideran positivo que un mismo profesor oriente la formación de un grupo durante todo proceso de aprendizaje en un área de conocimiento bajo el supuesto que facilita el avance, progresión y profundización en los contenidos. Además de ello, suponen conoce mejor a sus estudiantes y por consiguiente realiza un seguimiento más preciso y detallado. Para otros puede acontecer lo contrario, el profesor deja de lado temas que no son de su dominio, y hay estudiantes que no se adaptan a sus metodologías.

Esta discusión queda abierta, si bien es cierto que la continuidad de un profesor en el proceso formativo de los estudiantes puede llevar a que tanto profesor como alumno tengan un mayor conocimiento de cada uno, ello no parece garantizar un buen rendimiento académico, y por tanto no puede asumirse como una variable de éxito.

En cuanto a la ansiedad y género se encontró las niñas más ansiosas, resultado que concuerda con las investigaciones de Reali et al. (2015), Castro (2017), Agüero et al. (2017), un estudio realizado por la Universidad de Cambridge con estudiantes británicos de secundaria donde las niñas se mostraron más ansiosas que los niños (Álvarez, 2012), así como el efectuado con estudiantes españoles por Lozano (2013). Pero estos datos, como postulan Devine, Fawcett, Szücs y Dowker (2012) no deben tomarse como axiomáticos ya que hay muchos estudios en los cuales no se encontró diferencia de género y en otros se hallaron niveles más altos de ansiedad matemática en varones.

Al respecto Birgin, Baloğlu, Çatlığlu y Gürbüz (2010), sugieren que las diferencias en los resultados pueden deberse al uso diferenciado de pruebas para medir ansiedad matemática. Estudios realizados con Escala de ansiedad matemática de Fennema-Sherman, por Nortes y 
Nortes (2017) y Agüero et al. (2017), encontraron que las mujeres se muestran más ansiosas que los hombres.

Para una explicación más plausible de lo encontrado en esta investigación, se asume lo propuesto por Perina (2002) quien considera que no existe tal diferencia, sino que las mujeres, al responder pruebas reportan más episodios de ansiedad matemática que los hombres.

En lo correspondiente al objetivo general, la identificación de la relación entre ansiedad matemática y rendimiento académico en matemáticas, los resultados muestran la existencia de una correlación inversa, o sea que a mayor ansiedad menor rendimiento, resultados que coinciden con lo encontrado por Devine et al (2010) y Castro (2017), quienes evidenciaron que los estudiantes más ansiosos tenían peores notas. Estos resultados que se han encontrado en diversas investigaciones, llevaron a $\mathrm{Ma}$ y Xu (2004) a plantear las siguientes razones explicativas: a) ciertos niveles de ansiedad causarían bajos rendimientos escolares; b) son los bajos rendimientos los determinantes de la presencia de la ansiedad hacia las matemáticas; c) la ansiedad y el rendimiento en matemáticas están recíprocamente relacionados.

Teniendo en cuenta que el nivel de ansiedad arrojado por la prueba, muestra un nivel bajo de ansiedad y que el promedio académico es alto, de los anteriores presupuestos se acoge para explicar los resultados la que dice: "ciertos niveles de ansiedad causarían bajos rendimientos escolares", bajo el supuesto que el nivel de ansiedad encontrado es adaptativo, lo que le permite al estudiante no distraerse por consiguiente estar alerta, atento y no considerar las matemáticas como una actividad amenazante.

\section{Conclusiones}

Los datos dejan ver que la matemática generó reacciones de ansiedad baja en los escolares participantes en la investigación, que el nivel de ansiedad es mayor en las niñas así como la existencia de una correlación inversa entre rendimiento y ansiedad ya que en la medida que la ansiedad sube el rendimiento baja, dato coincidente con el encontrado en otras investigaciones como la realizada por Mato y Muñoz (2010), situación que muestra la influencia de factores afectivos en el aprendizaje de las matemáticas y lleva a proponerle al colegio la elaboración de un programa académico que fortalezca los estudiantes, especialmente a las niñas, en la parte motivacional y actitudinal de forma tal que modifiquen respuestas emocionales ante las matemáticas.

\section{Referencias}

Agüero, E., Meza, L., Suárez, Z. \& Schmidt, S. (2017). Estudio de la ansiedad matemática en la educación media costarricense. Revista Electrónica de Investigación Educativa, 19(1). DOI: https://doi.org/10.24320/redie.2017.19.1.849

Álvarez, R. (13 de julio de 2012). La ansiedad a las matemáticas afecta más a las niñas. Vital. Recuperado de https://rpp.pe/vital/expertos/ansiedad-hacia-las-matematicas-afecta-mas-a-lasninas-segun-estudio-noticia-501478

Ashcraft, M. \& Kirk, E. (2001). The relationships among working memory, math anxiety, and performance. Journal of Experimental Psychology General, 130(2), 224-237. DOI: $10.1037 / / 0096-3445.130 .2 .224$

Ashcraft, M. \& Moore, A. (2009). Mathematics anxiety and the affective drop in performance. Journal of Psychoeducational Aseasen, 27(3), 197-205. DOI: https://doi.org/10.1177/0734282908330580

Barrera, M., Gutiérrez, H., Lara, A. \& Villalpando, J. (2011). El rendimiento académico de las mujeres en matemáticas: análisis bibliográfico y un estudio de caso en educación superior en 
México. Revista Electrónica Actualidades Investigativas en Educación, 11(2). DOI: 10.15517/AIE.V11I2.10198

Birgin, O., Baloğlu, M., Çatlığlu, H. \& Gürbüz, R. (2010). An investigation of mathematics anxiety among sixth through eighth grade students in Turkey. Learning and Individual Differences, 20, 654-658. DOI: https://doi.org/10.1016/j.lindif.2010.04.006

Caciá, D., Reyes, M., Rosales, C. \& Marroquín, C. (2012). Factores que inciden en el rendimiento en matemática de niñas y niños del primer ciclo del nivel de educación primaria en escuelas públicas de Guatemala. Guatemala: Ministerio de Educación de Guatemala.

Caprile, M., Vallès, N., Potrony, J., Crèixams, C. \& Arasanz, J. (2008). El sesgo de género en el sistema educativo. Su repercusión en las áreas de Matemáticas y Tecnología en secundaria (THEANO). (Informe $\mathrm{N}^{\circ}$ 803-09-080-8). Barcelona: Instituto de la mujer. Recuperado de http://www.inmujer.gob.es/areasTematicas/estudios/estudioslinea2009/docs/elSesgoGenero.p df

Castro, G. (2017). La ansiedad y logros de aprendizaje en el área de matemática en estudiantes del quinto año de secundaria de la institución educativa San José de Nazareth. UGEL $\mathrm{N}^{\circ} 4$. Puente Piedra. 2016 (Tesis maestría). Universidad Cesar Vallejo. Lima. http://repositorio.ucv.edu.pe/bitstream/handle/UCV/5872/Castro_CGC.pdf?sequence=1\&isAl lowed $=\mathrm{y}$

Devine, A., Fawcett, K., Szücs, D. \& Dowker, A. (2010). Gender differences in mathematics anxiety and the relation to mathematics performance while controlling for test anxiety. Behavioral and Brain Functions, 8(33). DOI: https://doi.org/10.1186/1744-9081-8-33.

Dos Santos, J. \& Morale, A. (2012). Reversão de ansiedade à matemática: alguns dados da literatura. Psicologia em Estudo, 17(2), 317-327. DOI: http://dx.doi.org/10.1590/S141373722012000200015.

Fernández, R., Hernández, C., Prada, R. \& Ramirez, P. (2018). Dominio afectivo y prácticas

pedagógicas de docentes de Matemáticas: un estudio de revision. Revista Espacios, 39(23), 25 35.

Gamboa, R. (2012). ¿Equidad de género en la enseñanza de las Matemáticas? Revista Electrónica Educare, 16(1), 63-78.

González, J., Núñez, J., González, L., González, J. \& Roces, C. (2003). ¿Cómo explicar tanto fracaso en el aprendizaje de las matemáticas? Revista Galego-Portuguesa de Psicoloxía e Educación, 8(10), 350-358.

Guerrero, E. \& Blanco, L. (2004). Diseño de un programa psicopedagógico para la intervención en los trastornos emocionales en la enseñanza y aprendizaje de las matemáticas. Revista Iberoamericana de Educación, 34(2), 1-14. DOI: https://doi.org/10.35362/rie3422990

Hidalgo, S., Maroto, A. \& Palacios, A. (2004). ¿Por qué se rechazan las matemáticas? Análisis evolutivo y multivariante de actitudes relevantes hacia las matemáticas. Revista de Educación, 334, 75-95.

Jaggernauth, S. \& Jameson-Charles, M. (2010). Mathematics anxiety and the primary school teacher: an exploratory study of the relationship between mathematics anxiety, mathematics teacher efficacy, and mathematics avoidance. En M. Carmo (Ed. de la serie Educational Trends), Education Applications \& Developments Advances in Education (pp. 45-58) Recuperado

de https://uwispace.sta.uwi.edu/dspace/bitstream/handle/2139/12548/Sharon\%20Jaggernauth.pdf ?sequence $=1 \&$ is Allowed $=\mathrm{y}$

Linares, A. (2013). ¿Por qué somos tan malos en matemáticas? Recuperado de https://www.eltiempo.com/archivo/documento/CMS-13088961

Lozano, J. (12 de agosto de 2013). La ansiedad matemática de los alumnos españoles. El Mundo. Recuperado de https://www.elmundo.es/espana/2013/12/07/52a3004861fd3dd86b8b458f.html 
Ma, X. \& Xu, J. (2004). The causal ordering of mathematics anxiety and mathematics achievement: a longitudinal panel analysis. Journal of Adolescence, 27(2), 165-179. DOI https://doi.org/10.1016/j.adolescence.2003.11.003

Martínez, J. (2010). ¿Debe un profesor continuar con los mismos estudiantes de un grado a otro por varios años consecutivos en una institución educativa? Recuperado de http://viref.udea.edu.co/contenido/pdf/264-debe.pdf

Mato, M. \& Muñoz, J. (2010). Efectos generales de las variables actitud y ansiedad sobre el rendimiento en matemáticas en alumnos de educación secundaria obligatoria. Implicaciones para la práctica educativa. Ciencias Psicológicas, 4(1), 27-40.

Mendick, H. (2005). A Beautiful Myth? The Gendering of Being/Doing "Good at Maths". Gender and Education, 17(2), 203-219. https://doi.org/10.1080/0954025042000301465

Mesa, F. (2016). Algunos factores que influyen en los resultados de las pruebas estandarizadas y censales. Revista Redipe, 5(3), 136-145.

Ministerio de Educación Nacional. (2009). Escala de valoración nacional (Decreto No 1290). Recuperado de: https://www.mineducacion.gov.co/1621/articles187765_archivo_pdf_decreto_1290.pdf

Nortes, R. \& Nortes, A. (2017). Ansiedad, motivación y confianza hacia las Matemáticas en futuros maestros de Primaria. Números, 95, 77-92.

OECD (2019). Colombia - Country Note - PISA 2018 Results. Recuperado de https://www.oecd.org/pisa/publications/PISA2018_CN_COL_ESP.pdf

Palacios, A., Hidalgo, S., Maroto, A. \& Ortega, T. (2013). Causas y consecuencias de la ansiedad matemática mediante un modelo de ecuaciones estructurales. Enseñanza de las ciencias, 31(2), 93-111.

Pérez-Tyteca, P. (2012). La ansiedad matemática como centro de un modelo causal predictivo de la elección de carreras. (Tesis doctoral). Universidad de Granada, España. https://hera.ugr.es/tesisugr/2108144x.pdf

Pérez-Tyteca, P., Castro, E., Segovia, I., Castro, E., Fernández, E. \& Cano, F. (2007). Ansiedad matemática de los alumnos que ingresan en la Universidad de Granada. Investigación en Educación Matemática, 11, 171-180.

Pérez-Tyteca, P., Monje, J. \& Castro, E. (2013). Afecto y matemáticas. Diseño de una entrevista para acceder a los sentimientos de alumnos adolescentes. Avances de Investigación en Educación Matemática, 4, 65-82. DOI: https://doi.org/10.35763/aiem.v1i4.55

Perina, K. (2002). Sum of All Fears. Recuperado de https://www.psychologytoday.com/us/articles/200211/sum-all-fears

Radovic, D. (2018). Diferencias de género en rendimiento matemático en Chile. Revista Colombiana de Educación. 74. 221-242.

Reali, F., Maldonado, C. \& Jiménez, W. (2015). Ansiedad a las matemáticas y bajo desempeño: ¿Son las niñas y los estudiantes de últimos años escolares los más afectados? Sexteto, 6. Recuperado de https://sextante.uniandes.edu.co/index.php/ejemplares/sextante6/horizontes/ansiedad-a-las-matematicas-y-bajo-desempeno-son-las-ninas-y-los-estudiantes-deultimos-anos-escolares-los-mas-afectados

Reyes, L. (1984). Affective variables and Mathematics education. The Elementary School Journal, 84(5, 558-581.

Richardson, F. \& Suinn, R. (1972). The Mathematics Anxiety Rating Scale: Psychometric data. Journal of Counseling Psychology, 19(6), 551-554.

Rius, M. (21 de mayo de 2015). ¿Por qué muchos estudiantes odian las matemáticas? Vanguardia.

Recuperado

de www.lavanguardia.com/vida/20150521/54431772174/estudiantes-odian-matematicas.html

Rivas, F. (1997). El proceso de enseñanza/aprendizaje en la situación educativa. Barcelona: Ariel Planeta. 
Sánchez, C. (2017). Las mediciones masivas: una producción política de sentidos y significados sobre los sistemas educativos. Sophia, 13(1), 64-74.

Sánchez, J., Segovia, I. \& Miñán, A. (2011). Exploración de la ansiedad hacia las matemáticas en los futuros maestros de educación primaria. Profesorado, 15(3), 297-312.

Serrano, R. \& Sánchez, G. (2017). La especificidad de la ansiedad matemática en estudiantes mexicanos de bachillerato. Acta Latinoamericana de Matemáticas Educativa, 30, 178-187.

Contribución de los autores: a) Concepción y diseño del trabajo; b) Adquisición de datos; c) Análisis e interpretación de datos; d) Redacción del manuscrito; e) revisión crítica del manuscrito.

G.V.A. ha contribuido en a,b,c,d,e; T.Y.A.A. en a,b,c,d,e; W.J.T.C. en a,b,c,d,e.

Editora científica responsable: Dra. Cecilia Cracco 